\title{
The Realization of the Geological Image 3d Reconstruction
}

\author{
Du Cheng \\ College of Electric and Information \\ Engineering,Southwest University for Nationalities \\ Chengdu, China
}

\author{
Leng Biao \\ School of Civil Engineering, Southwest Jiaotong \\ University, Chengdu,China
}

\begin{abstract}
Computer vision theory, the 3d object reconstruction is always a very important research subject. Most humans are attained through the visual perception of information, but in the real world, people think it is 3d information, how to better performance, the $3 \mathrm{~d}$ information, to be one of the hot topics of attention. 3d reconstruction application in image processing system also includes the application of image processing technology. This application is mainly manifested in the process of graphic artificial processing. This article through to the geological image building, the coordinates of triangles algorithm of image reconstruction algorithm, using OpenGL transform operation realize $3 \mathrm{~d}$ reconstruction image. Geological by computer image processing, 3d visualization of geological image. With the result feedback to geological construction management and design, in order to improve the geology observation precision, at the same time make the geological observation data preservation and effective utilization of possible.
\end{abstract}

Keywords-Image division Imagery processing, 3D, image recognition

\section{THE INTRODUCTION}

$3 \mathrm{~d}$ reconstruction is a multi-disciplinary comprehensive technology, it combines computer technology, computer graphics, image processing technology, the information synthesis technology, display technology, and many other high and new technology in a body. In recent years, because of the increase in computing power of the computer technology rapidly expanding the allocation of memory, disk space, network enhancements, many important graph generating and image processing algorithms are available hardware implementation, accelerated dramatically. Therefore, the rapid development of $3 \mathrm{~d}$ reconstruction technology has become possible. Foreign research on $3 \mathrm{~d}$ reconstruction most countries belong to Japan, followed by the United States and Britain. Developed countries started early, the research also more deeply. 3d reconstruction application field is very wide, almost involves all areas of natural science and engineering technology.

In the geological mining stage will produce a series of images, the images with relatively spatial relations. In these images are processed, able to correct for all kinds of the extract of texture characteristic data and images.
Finally, 3d reconstruction, these features show the three-dimensional structure.

On this basis, the user must be able to correctly deal with the image, to set the right of the image attributes, providing the necessary process information. Such as image file list file read and write operations, set the current image, maintaining the current image attributes, view the current image of the target object parameter data. Print the current image.

Set the current image, can according to the characteristics of the image to image processing operations, such as gray processing, color processing, binary image processing, texture image processing, etc. Uncertainty because of the complexity of the actual image and acquisition process, fully automatic image processing also has certain difficulty, at the same time, because the domestic haven't similar system for reference, also requires us to make constant adjustments in the later work. For this reason, also must add the necessary way of manual processing, to make up for the inadequacy of automatic image processing, make both supplement each other, play to their respective advantages, to achieve the best treatment effect.

In the completion of the process of image processing, image of the target object will be extracted. After the target object to quantitatively describe will form feature vector, vector space. All of these have a feature vector to the target object will show some characteristics in the vector space, these characteristics are further processing can be completed clustering, get the target of further information.

After completing the whole process of image processing, will be $3 \mathrm{~d}$ restructuring, all of the target object that has certain characteristics of the target correctly recombinant, finally formed a complete $3 \mathrm{~d}$ structure. 


\section{THE CLASSIFICATION OF 3D RECONSTRUCTION}

\section{TECHNOLOGY}

Three-dimensional reconstruction and display is basic and important part of visualization. People in the study on the algorithm of $3 \mathrm{~d}$ reconstruction and drawing process, has been committed to solve the problem of two aspects: one is the effect of $3 \mathrm{~d}$ reconstruction, i.e. reconstruction results of truth and visibility, it requires that the reconstruction results can be real and objectively reflects the characteristics of object structure in three dimensions and details; Two is the speed of the $3 \mathrm{~d}$ drawing, i.e. spatial geometry information data which are obtained by body data in a certain way of $3 \mathrm{~d}$ rendering time projected onto a computer screen.

\section{A. the surface rendering}

This is a traditional method, first of all to segmentation of fault data, extract the interested in the surface of the object information, constructed by the three dimensional space data field among geometric primitives (e.g., surface, plane, etc.), and then from the traditional computer graphics techniques such as clipping and geometry transform, according to the light, light and shade model picture drawing after blanking and rendering, image display.

\section{B. volume rendering}

Volume rendering method is also called the direct volume rendering method, its processing of $3 \mathrm{~d}$ data, is not between tectonic geometry primitives, but directly from $3 \mathrm{~d}$ data field to generate two-dimensional image on the screen, called the volume rendering algorithm, or direct volume rendering algorithm. It is developing rapidly in recent years a method of $3 \mathrm{~d}$ data field visualization in this method, mapping is omitted in this step, but directly to draw after refining and processing the data, to generate $2 \mathrm{~d}$ images. This algorithm can generate $3 \mathrm{~d}$ data field of the whole image, including every detail, and has advantages of high image quality, easy to parallel processing. The main problem is that a large amount of calculation, and difficult to use traditional drawing graphics hardware implementation, and thus a longer computation time.

\section{The implementation of 3 d reconstruction system}

We reconstruction of three-dimensional stereoscopic structure. From the longitudinal view, is the arrangement of a series of plane image, from the horizontal view, is a single cross section image. For each image, it first has a its own external boundary characteristics, it also has its own characteristics and the outer boundary boundary characteristics. So this is an internal circulation data structure.

specific data structures are defined as follows:

Header: [fileheader] - start tag

Version $=1.0--$, the version number

Slicenum $=\mathrm{N}$ - section number

Endheader - end tag

The data structure:

[sliceNI] - cut the ith slice
Objnum $=\mathrm{J}-$ section of the ith section contains the number of pixels

[sliceNI - J] - section of the ith slices first J a pixel tags

Edgenum $=\mathrm{K}$ - section of the ith section of the first kind of the boundary of the pixel number $\mathrm{J}$

[sliceNI - J - K] - section of the section I of the J pixels of the $\mathrm{K}$ boundary markers

Pointnum $=\mathrm{M}$ - the first section of the ith sliced $\mathrm{J}$ a pixel contains the number of points the first $\mathrm{K}$ boundary

The name of the ImageFile - the current import section images

ImageSize - the current into the size of the cross section image

PostLink did - the current section and its subsequent target of connection, the default value is 1 , if greater than zero, has said it connects to the target in the later section of the it has

PreLink did - the current section connected to the section in the preamble of its target, the default value is 1 , if greater than zero, has said it connects to the target in the cross section of the program before it has

PtI $(0<\mathrm{I}<\mathrm{M})=\mathrm{X} \mathrm{Y} \mathrm{Z}$, the coordinates of the target object

\section{D. $3 d$ reconstruction algorithm}

Is equipped with the adjacent two layers, which corresponding to the target object and Obj1 Obj2, their contour feature point sets some episodes of Obj1 for $\mathrm{M}$, the number of points Obj2 for $\mathrm{N}$. Obj1 Point 1 representative point set, Point 2 represent Obj2 point set, Obj1Pi represent a specific point of the Obj1, specific point in the Obj2Pj representative Obj2, Point $1=\{\mathrm{Obj} 1 \mathrm{Pi}$ $\mid 0<\mathrm{I}<\mathrm{M}\}$, Point $2=\{\mathrm{Obj} 2 \mathrm{Pj} \mid 0<\mathrm{j}<\mathrm{N}\}$, in clockwise order, the steps of algorithm are as follows:

1) the initial triangles. The selection of the first two points

First choose the starting point for the first goal, do not break general, assuming $\mathrm{Obj} 1 \mathrm{P} 0$ for target a first, computing Obj1P0 to second all the points in the target object distance, the statistics to the distance of the minimum point, assumed Obj2Pmin, therefore, set Obj1Pmin as the second, the starting point of the target object.

\section{2) the selection of initial triangles. The third point}

So connected Obj1P0 and Obj1Pmin, take both the halfway Point of the line segment, set the Point to Point, then calculate Point to two target first two points of the adjacent Point distance, that is

Obj1P1 and the distance to the Obj2Pmin +1 , if to Obj1P1 shortest distance, is taking

Obj1P1 point for triangles. The third point, if the distance to $\mathrm{Obj} 2 \mathrm{P} \min +1$ point, the shortest is taking

Obj2P min +1 point for triangles. The third point, given that the shortest distance Obj2P min +1 , start-up of the triangles as (Obj1P0) (Obj2Pmin) (Obj2P min + 1). Thus the initial triangles is constructed.

\section{3 ) the creation of a subsequent triangles}

Initial triangles constructed by subsequent triangles, firstly, the initial triangles of the adjacent side, such as according to the above assumptions, the neighborhood for 
the $(\mathrm{Obj} 1 \mathrm{P} 0)(\mathrm{Obj} 2 \mathrm{P} \min +1)$, the same rules, then select the midpoint of the line segment, and to calculate the midpoint (Obj1P0) and (Obj2P min +1$)$ distance of two adjacent points, see which neighboring points distance recently, and so on, on a subsequent triangles are constructed.

\section{4) the end of the triangular structure}

As these points according to the law has been proceed, but due to the different target object have different points, therefore, when the construction to proceed, must be finished in judgment. If the target object in a point circulation point-to-point $\mathrm{Obj} 1 \mathrm{P0}$ or $\mathrm{Obj} 2 \mathrm{P} \mathrm{min}$, the reconstruction jobs disappear.

\section{THE CREATION OF A 3D SCENE}

In front of the image processing and $3 \mathrm{~d}$ reconstruction, we must coordinate space of their definition, therefore, we define the $2 \mathrm{~d}$ coordinate system and $3 \mathrm{~d}$ coordinate system. In image processing, we introduced the concept of layers, in $3 \mathrm{~d}$ reconstruction, we define triangular subdivision for the rules. Next, we according to the structure characteristics of tunnel section of analysis, established the realization of the data structure of $3 \mathrm{~d}$ reconstruction, this part also is the key that we consider and deal with.

Coordinates definition: this system needs to be completed 2d image processing and $3 \mathrm{~d}$ graphics. Moreover, these data between internal relevance. In order to be able to deal with these two approaches under the coordinates of the corresponding relationship. In this first to define the coordinates of the space required. $2 \mathrm{~d}$ coordinates definition: $2 \mathrm{~d}$ coordinates in the image processing using cartesian space rectangular coordinate.

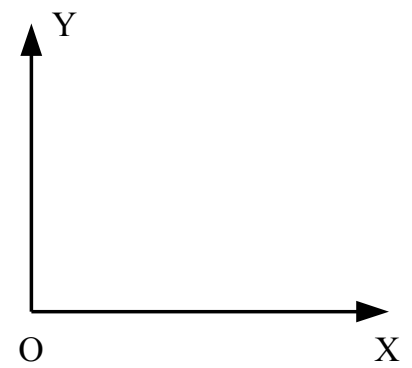

Figure 1. $2 \mathrm{~d}$ coordinates are defined

$3 \mathrm{~d}$ coordinate to define the $3 \mathrm{~d}$ coordinate is used the cartesian space rectangular coordinate, definition of the direction of the tunnel for the $\mathrm{Z}$ axis is the direction, and the initial vertical constraints; The $\mathrm{Y}$ axis is perpendicular to the horizontal plane direction; $\mathrm{X}$ axis perpendicular to the $\mathrm{Y}$, and $\mathrm{Z}$ for the constraints in the face of it, the direction is referred to in the right direction when level raised his right hand.

A tunnel in the mining process can form a cavity, the cavity is a tunnel wall (the outline of the figure in red line). At the same time, every time in the process of image acquisition is the process of a series of constraints to obtain data of tunnel (blue oval curve).

$3 \mathrm{~d}$ reconstruction of the system is using triangular reconstruction algorithm, in order to create the tunnel cross section of three-dimensional nuclear model, we must first have to create a $3 \mathrm{~d}$ scene environment. A series of triangles formed a series of grid objects, so we need to add the grid object to scene space, but the grid object is composed of vertex data and triangles together, therefore, to the number of vertices and the number of triangles. The number of vertices and triangles to add to the scene. Finally, to compute adjacent vertex, each facet normals, each vertex normal, in the program, we specially compiled class CSceneGraph3d, as to the operation of the 3-d scene space. The implementation process of specific creation scenario is as follows:

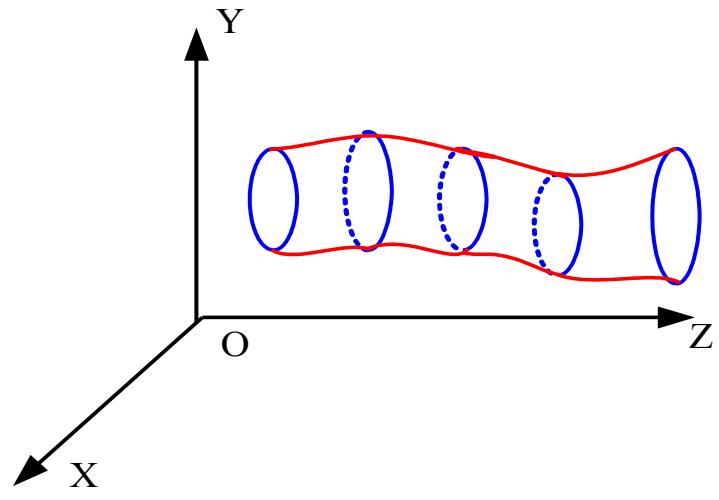

Figure 2. 3d coordinates are defined

$3 \mathrm{~d}$ reconstruction of the system is using triangular reconstruction algorithm, in order to create the tunnel cross section of three-dimensional nuclear model, we must first have to create a $3 \mathrm{~d}$ scene environment. A series of triangles formed a series of grid objects, so we need to add the grid object to scene space, but the grid object is composed of vertex data and triangles together, therefore, to the number of vertices and the number of triangles. The number of vertices and triangles to add to the scene. Finally, to compute adjacent vertex, each facet normals, each vertex normal, in the program, we specially compiled class CSceneGraph3d, as to the operation of the 3-d scene space. The implementation process of specific creation scenario is as follows:

CSceneGraph3d * pSceneGraph;

PSceneGraph = new CSceneGraph3d; / / create a scenario $\mathrm{CMesh} 3 \mathrm{~d} * \mathrm{pMesh}=$ new $\mathrm{CMesh} 3 \mathrm{~d} ; / /$ create a mesh object

PSceneGraph - > Add (pMesh); / / add grid objects to the scene

/ / set the vertex of a mesh number and the number of triangles

PMesh - > m_ArrayVertex. SetSize (dwVertexNum);

PMesh - > m_ArrayFace. SetSize (dwFaceNum);

/ / to extract the vertices and triangle surface object

CArray $3 \mathrm{~d}<$ CVertex $3 \mathrm{~d}>*$ pArrayVertex $=\&($ pMesh $->$ m_ArrayVertex);

CĀrray3d $<$ CFace3d $>*$ pArrayFace $=\&($ pMesh $->$ m_ArrayFace);

/ / add the vertices

PArrayVertex - > SetAt (I, new CVertex3d (fVertext [0], fVertext [1], fVertext [2]));

/ / add triangle surfaceCVertex $3 \mathrm{~d} * \mathrm{p} 1=$ pArrayVertex - > GetAt (nFace [0]).

CVertex3d * p2 = pArrayVertex - $>$ GetAt (nFace [1]).

CVertex $3 \mathrm{~d}^{*} \mathrm{p} 3=$ pArrayVertex $->$ GetAt (nFace [2]). 
CFace $3 \mathrm{~d} *$ pFace $=$ new CFace $3 \mathrm{~d}$;

PFace - > Set (p1, p2, p3);

PArrayFace - $>$ SetAt (I, pFace);

/ / on the surface of the adjacent vertex, each method to, each vertex normals

PMesh - > BuildAdjacency ();

PMesh - > CalculateNormalPerFace ();

PMesh - > CalculateNormalPerVertex ();

Deletet pSceneGraph; / / delete existing scenario

\section{TUNNEL 3D RECONSTRUCTION AND 3 D PREDICTION}

In order to know the construction of the tunnel geological tectonic situation, it is necessary to simulate the $3 \mathrm{~d}$ reconstruction of the tunnel, through the reconstruction of $3 \mathrm{~d}$ tunnel model from different Angle to observe the geological condition of tunnel. $3 \mathrm{~d}$ reconstruction using OpenGL 3d modeling technology. The Open is the full name of OpenGL Graphics Libaray, is a $3 \mathrm{~d}$ Graphics and model library. It is a new generation of $3 \mathrm{~d}$ graphics industry standard. OpenGL is not only a graphics library, it is an Application program Interface (Application Programming Interface).

Need to pay attention to in the process of $3 \mathrm{~d}$ reconstruction is how to build the block boundary constraints of adjacent images of the same strata corresponding relation, only the corresponding relationship to establish correct, to restore the accurate three-dimensional models. In the system by pattern recognition algorithm will be that of the strata boundary constraints of adjacent images, eventually establish the constraints in image sequence strata corresponding relation. At the same time, considering the situation, there may be predicted using the algorithm of pattern recognition especially rock fault occurred, make the rock that should be associated with no connection, but should not be associated with rock. Way, therefore, need to use manual intervention to the actual rock target in the adjacent images corresponding relation changes are relatively. Fig . 3 is constraints according to the sequence established three-dimensional model of tunnel.

Fig .4, the most outside the wireframe represents the outer contour of the tunnel, can from different angles to look at the $3 \mathrm{~d}$ model of the tunnel.

\section{TUNNEL THREE-DIMENSIONAL PREDICTION}

According to tunnel three-dimensional model, can only be observed in the tunnel has excavation section of the geological structure, can only play a guiding role to a certain extent on tunnel construction. Contractor usually also want to be able to understand the tunnel surrounding and geological structure of the front not tunnel excavation. And automatically by the computer for tunnel construction and tunnel surrounding ahead to forecast and the forecast results is displayed with $3 \mathrm{~d}$ model and can go a step further to provide reference for tunnel construction. This will have a lot of direct effect of tunneling construction, make more reasonable construction measures in construction process, make construction process more secure.

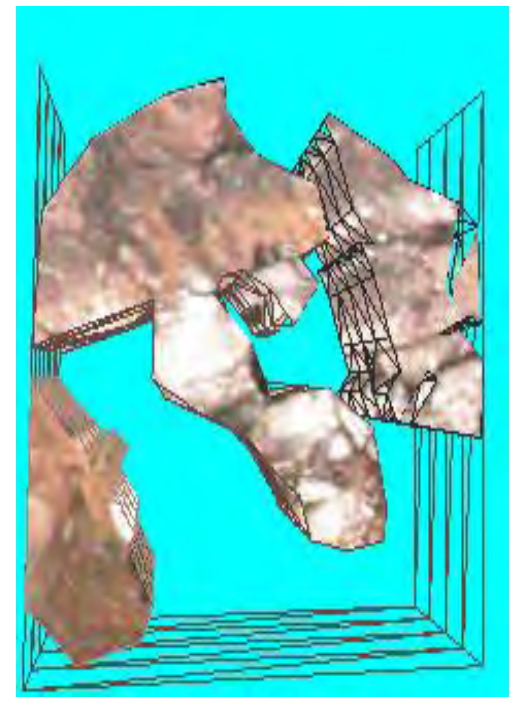

Figure 3. tunnel three-dimensional model

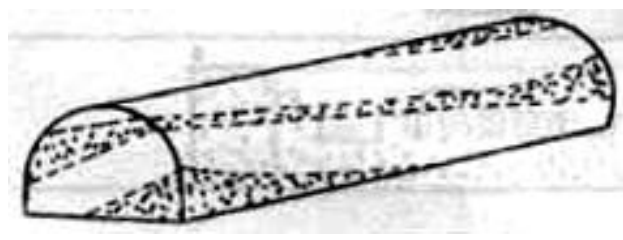

Figure 4. tunnel geological 3 d sketch

\section{THE CONCLUSION AND PROSPECT}

In computer vision, three-dimensional and two-dimensional projection images, a transformation relationship between and the relationship is determined by the camera imaging geometric model. This paper implements a method to reconstruct $3 \mathrm{~d}$ image, for future professionals can make a correct interpretation of original data, and get the position of mineral deposits in existence, and the size of the storage, and other important information. We can see that it is of great economic and social benefits.

\section{ACKNOWLEDGEMENT}

This work is Supported by the Fundamental Research Funds for the Central Universities, Southwest University for Nationalities (2014NZYQN09)

\section{REFERENCE}

[1] Donald Hearn \& m. Pauline Baker.Computer Graphics. Publishing House of Electronics Industry, 2002

[2] Leng Biao;Qiu Wenge;Du Cheng,"Tunnel Three-Dimensional Modeling Research Based On Digital Image Processing",2008 International Conference On Computer Science And Software Engineering,IEEEPress,Dec.2008,pp:887-890,Vol1,Doi:10.1109/C sse. 2008.301

[3] chun-lin zhou, zhu, Li Xiaojun. "New Austrian method tunnel construction constraints infrared camera and image processing", 
Journal of rock mechanics and engineering. 2008.6, 27pp: 3166-3172,

[4] Ye Ying, wang. "Tunnel geological information digital logging identification technology research" Journal of Beijing jiaotong university. 2007.2,pp 59-62.

[5] LangRui. Digital image processing. Beijing hope electronic publishing house, 2002

[6] Rafael c. Gonzalez \& Richard e. Woods, Digital Image Processing. Publishing House of Electronics Industry, 2003

[7] Bin Abdul Rashid, N. Taib, M.N. ; Lias, S. ; Sulaiman, N. ; Murat, Z.H. ; Kadir, R.S.S.A.” Enhancement Techniques for Tuberculosis Slide Images: A Review", International Conference on Biomedical Engineering,,IEEEPress,Feb.2012,pp:527-531,DOI:10.1109/ICoB E.2012.6179074
[8] Xiaosheng Yu, Chengdong Wu,"A New Digital Image Restoration Model Based on Local Constraints", 2012 International Conference on Industrial Control and Electronics Engineering (ICICEE), IEEE Press, Aug,2012,pp:456-459, DOI:10.1109/ICICEE.2012.127

[9] Qiushi Bai, Limeng Zhao ; Yunzhou Zhang ; Zixin Qi," Research of automatic recognition of digital meter reading based on intelligent image processing", Computer Engineering and Technology (ICCET), 2010 2nd International Conference on , IEEE Press, April 2010, Vol5,pp: 619-623, DOI:10.1109/ICCET.2010.5486243

[10] Hu Shijie,Huang Qi," Research on automobile sketch design based on image cognition model" Image and Signal Processing (CISP), 2011 4th International Congress on, IEEE Press, Oct. 2011, Vo3,pp: 1549 - 1552, DOI: 10.1109/CISP.2011.610049 